\title{
METODOLOGÍA DIAGNÓSTICA ANTE LA RECIDIVA BIOQUÍMICA DESPUÉS DE PROSTATECTOMÍA RADICAL.
}

\author{
Argimiro Collado Serra y Eduardo Solsona Narbón.
}

Servicio de Urología. Instituto Valenciano de Oncología (IVO). Valencia. España.

\begin{abstract}
Resumen.- El cáncer de próstata es uno de los principales problemas de salud de la población masculina. La prostatectomía radical ha demostrado una excelente tasa de curación a largo plazo. No obstante, de forma global, un $25 \%$ de los pacientes intervenidos experimentarán una elevación del PSA durante los 15 años de seguimiento. En general, el valor de PSA asociado con un mayor riesgo de progresión clínica y por lo tanto, merecedor de ser establecido como punto de corte para una recidiva bioquímica es de $0.4 \mathrm{ng} / \mathrm{ml}$. Una vez detectada la recidiva bioquímica, el dato clínico más importante será determinar si la recidiva clínica se va a producir a nivel local o sistémico, ya que ello de-
\end{abstract}

terminará el tratamiento a realizar. Los principales parámetros que ayudan a diferenciar entre una y otra son los siguientes: intervalo de tiempo hasta el incremento del PSA, la velocidad del PSA, el tiempo de doblaje del PSA (PSADT), el estadio patológico y el Gleason de la pieza quirúrgica. Las posibilidades de tratamiento ante el fracaso bioquímico de una PTR están sometidas todavía a debate. No obstante, actualmente se considera que los pacientes con una recidiva bioquímica sin evidencia radiológica de metástasis a distancia son candidatos ideales para el tratamiento local con radioterapia.

Palabras clave: Cáncer de próstata. Recidiva bioquímica. Prostatectomía radical. PSA.

Summary.- Prostate cancer is one of the main health problems of the male population. Radical prostatectomy has demonstrated to have an excellent long-term cure rate. Nevertheless, globally, a 25\% of the operated patients will suffer a PSA increase over 15 years of follow-up. Generally, the PSA value associated with a higher risk of clinical progression, that may be established as the cut point for biochemical recurrence is $0.4 \mathrm{ng} /$ $\mathrm{ml}$. Once biochemical recurrence is diagnosed, the most important clinical data is to determine if clinical recurrence is going to be local or systemic, because it will determine treatment. Main parameters helping to differentiate between one and another are: time interval to PSA increase, PSA velocity, PSA doubling time IPSADT), pathologic stage and specimen's Gleason's score. The possibilities of treatment of biochemical failure after radical prostatectomy are under debate. Nevertheless, it is currently considered that patients with biochemical 
recurrence without radiological evidence of distant metastases are ideal candidates for local treatment with radiotherapy.

Keywords: Prostate cancer. Biochemical recurrence. Radical prostatectomy. PSA.

\section{INTRODUCCIÓN}

El cáncer de próstata es uno de los principales problemas de salud de la población masculina, ya que es la neoplasia más frecuente. En 1999, supuso el $31 \%$ de todos los tumores diagnosticados en varones (1). Se calcula que en Europa se diagnostican 2.6 millones de nuevos casos al año, donde constituye el $11 \%$ de todos los cánceres masculinos, y es el responsable del $9 \%$ de todas las muertes por cáncer (1).

En líneas generales, y al margen de los programas de screening, sólo el $55 \%$ de los tumores están clínicamente localizados en el momento del diagnóstico (1). El manejo habitual del PSA ha supuesto un cambio radical en el diagnóstico y seguimiento de pacientes con cáncer de próstata. Así, se ha constatado en los últimos años un descenso en la edad media de los pacientes diagnosticados y un descenso en la proporción de enfermedad metastásica (2).

La prostatectomía radical ha demostrado conseguir una excelente tasa de curación a largo plazo, con una supervivencia libre de metástasis del $82 \%$ a los 15 años de seguimiento en pacientes con estadios localizados. A pesar de que la prostatectomía radical (PTR) y la radioterapia (RT) prostática están más que establecidas como tratamiento curativo, existe un no desdeñable riesgo de recidiva tumoral. Durante los 10 primeros años de seguimiento, entre un 27 y un $53 \%$ de los pacientes desarrollarán una recidiva local o metástasis a distancia, y entre un 16 y un $35 \%$ de los pacientes realizarán un segunda línea de tratamiento dentro de los 5 años del tratamiento inicial (1).

\section{PSA TRAS TRATAMIENTO RADICAL INICIAL}

A pesar de los buenos resultados oncológicos de la prostatectomía radical un $25 \%$ de los pacientes intervenidos experimentarán una elevación del PSA durante los 15 años de seguimiento (3).
Tras una prostatectomía radical, al resecarse todo el tejido prostático, el PSA sérico debe reducirse hasta límites indetectables a las $4-6$ semanas de la cirugía (4). Sin embargo, el tiempo medio hasta alcanzar el PSA nadir después de la radioterapia radical puede llegar hasta los 18 meses, incluso más después de la braquiterapia (2).

Tradicionalmente se ha asociado el fallo del tratamiento con la demostración de un fallo local (tacto rectal y biopsia) o el desarrollo de metástasis a distancia. Sin embargo, hoy en día el fallo del tratamiento se tiende a asociar al aumento del PSA (recidiva bioquímica), ya que es difícil que con un seguimiento de 5 años, un paciente desarrolle una recidiva clínica sin un aumento concomitante del PSA (5). El problema estriba en reconocer qué nivel de PSA se considera fracaso o recidiva bioquímica (BCR). Aunque hay muchos factores implicados, va a depender principalmente del tratamiento radical que se haya aplicado y de la sensibilidad diagnóstica que deseemos alcanzar (2).

\section{DEFINICIÓN DE RECIDIVA BIOQUÍMICA TRAS PROSTATECTOMÍA RADICAL}

Tal como se ha comentado previamente, del punto de corte inicial dependerá la tasa de pacientes que durante el seguimiento experimentarán una elevación del PSA y una posterior recidiva clínica. Si bien la utilidad del PSA en el seguimiento está clara, la relación con la recidiva clínica y la mortalidad cáncer especifica todavía continua sin tener explicación (2), ya que muchos pacientes con elevación del PSA durante el seguimiento permanecerán libres de recidiva clínica sin ningún tratamiento adicional (5).

Amling y cols. estudian 2.782 pacientes intervenidos de PTR por cáncer de próstata localizado (cT1-T2) (6). Se realizó el seguimiento mediante PSA cada 3 meses durante el primer y segundo año, cada 6 meses hasta los 5 años de la cirugía, y después de forma anual. La mediana de seguimiento de la serie fue de 6.3 años. La sensibilidad en la detección de la progresión clínica depende, lógicamente, del punto de corte establecido. Así, una vez detectada la recidiva del PSA, el subsiguiente incremento del PSA se daba en un $49 \%, 62 \%$ y $72 \%$ en pacientes con PSA de $0.2,0.3$ y $0.4 \mathrm{ng} / \mathrm{ml}$, respectivamente. Este dato indicaba que sólo la mitad de los pacientes con un PSA superior a $0.2 \mathrm{ng} / \mathrm{ml}$ progresaban durante el seguimiento. Para ellos el valor de PSA asociado con un mayor riesgo de progresión clínica y por lo tanto, merecedor de ser establecido como punto de corte para una $B C R$, era de $0.4 \mathrm{ng} / \mathrm{ml}$ (Tabla I). 
TABLA I. ESTIMACIÓN TRAS 3 AÑOS DE SEGUIMIENTO DEL PORCENTAJE DE PACIENTES CON INCREMENTO DEL PSA DE ACUERDO CON EL PRIMER PSA DETECTABLE.

\begin{tabular}{|l|c|c|c|}
\hline PSA inicial (ng/ml) & $\begin{array}{c}\text { Número de pacientes con } \\
\text { este PSA inicial }\end{array}$ & $\begin{array}{c}\text { Porcentaje de pacientes } \\
\text { con posterior incremento } \\
\text { del PSA }\end{array}$ & $\begin{array}{c}\text { Porcentaje de pacientes } \\
\text { con posterior incremento } \\
\text { del PSA o tratamiento }\end{array}$ \\
\hline $0.20-0.29$ & 773 & 49 & 50 \\
\hline $0.30-0.39$ & 450 & 72 & 65 \\
\hline $0.40-0.49$ & 323 & 62 & 79 \\
\hline $0.50-0.59$ & 279 & 60 & 67 \\
\hline $0.60-0.69$ & 172 & 77 & 84 \\
\hline $0.70-0.79$ & 175 & 75 & 88 \\
\hline $0.80-0.89$ & 137 & 76 & 87 \\
\hline
\end{tabular}

Otros autores consideran puntos de corte más bajos. Freedland y cols. evalúan 358 pacientes intervenidos de PTR. Para una nivel de corte de PSA postoperatorio entre $0.11 \mathrm{ng} / \mathrm{ml}$ y $0.2 \mathrm{ng} / \mathrm{ml}$, el riesgo de progresión del PSA fue del $64 \%$ al año y del $93 \%$ a los 3 años de seguimiento. Sin embargo, para pacientes con un PSA postoperatorio entre 0.21 y $0.3 \mathrm{ng} / \mathrm{ml}$, el riesgo de progresión fue del $86 \%$ al año y del $100 \%$ a los 3 años (7). En el análisis multivariante, el PSA preoperatorio y el Gleason fueron para estos autores las dos únicas variables clínicas que tuvieron capacidad predictiva para la recidiva bioquímica, de forma independiente al valor de corte utilizado (Tabla II y III).

Stephenson y cols. evaluan 12 definiciones de progresión bioquímica en 4035 pacientes intervenidos entre 1983 y 2003 (8). Con un seguimiento medio de 49 meses, 118 pacientes desarrollaron metástasis a distancia. Para cada definición de $B C R$ se realizó un análisis multivariante de Cox, donde el valor estadístico R2 se utilizó para determinar la definición que mejor predecía la aparición de metástasis a distancia. La supervivencia libre de enfermedad a

TABLA II. RIESGO DE RECIDIVA BIOQUIMMICA EN FUNCIÓN DEL PUNTO DE CORTE DE PSA ESTABLECIDO COMO DEFINICIÓN DE LA MISMA (7).

\begin{tabular}{|l|c|c|}
\hline $\begin{array}{l}\text { Punto de corte del PSA } \\
\text { (ng/ml) }\end{array}$ & $\begin{array}{c}\text { Porcentaje de pacientes con recidiva } \\
\text { bioquímica a los 3 años (\%) }\end{array}$ & $\begin{array}{c}\text { Porcentaje de pacientes con recidiva } \\
\text { bioquímica a los 5 años (\%) }\end{array}$ \\
\hline$>0.01$ & $36(30-42)^{*}$ & $55(49-2)$ \\
\hline$>0.1$ & $25(21-31)$ & $43(36-50)$ \\
\hline$>0.2$ & $21(16-26)$ & $39(33-47)$ \\
\hline$>0.3$ & $19(14-24)$ & $33(27-40)$ \\
\hline$>0.4$ & $16(12-21)$ & $26(21-33)$ \\
\hline$>0.5$ & $14(10-19)$ & $23(18-30)$ \\
\hline
\end{tabular}

* Intervalo de confianza 95\% 
TABLA III. PORCENTAIE DE PACIENTES CON INCREMENTO DEL PSA A 3 AÑOS DE SEGUIMIENTO DE ACUERDO CON EL PRIMER PSA DETECTABLE (7).

\begin{tabular}{|l|c|c|}
\hline PSA (ng/ml) & Pacientes $(\mathbf{n})$ & $\begin{array}{c}\text { Riesgo de incremento PSA a los 3 } \\
\text { años (\%) }\end{array}$ \\
\hline $0.01-0.1$ & 73 & $67(46-87)^{*}$ \\
\hline $0.11-0.2$ & 34 & $93(74-99)$ \\
\hline $0.21-0.3$ & 26 & $100(87-100)$ \\
\hline $0.31-0.4$ & 29 & $84(61-97)$ \\
\hline $0.41-0.5$ & 11 & $89(61-99)$ \\
\hline
\end{tabular}

* Intervalo de confianza $95 \%$

los 10 años estaba entre el $79 \%$ de los casos si se utilizaba los criterios de la ASTRO (9) y el $56 \%$ si se utilizaba como BCR la presencia de un valor de PSA superior a $0.2 \mathrm{ng} / \mathrm{ml}$. De acuerdo al análisis multivariante y una vez controladas las variables clínicas y la presencia de tratamientos de segunda línea, la mejor definición para detectar metástasis a distancia era la presencia de un PSA superior a $0.4 \mathrm{ng} / \mathrm{ml}$ seguido de una elevación posterior durante el seguimiento.

De acuerdo a la "Guías Clínicas de la Asociación Europea de Urología", en un paciente intervenido de prostatectomía radical, dos valores consecutivos de PSA superior a $0.2 \mathrm{ng} / \mathrm{ml}$ representan una recidiva bioquímica (1).

Ward y cols. estudian 3903 varones sometidos a prostatectomía radical por un cáncer de próstata localizado (10). El seguimiento medio de la serie fue de 8.8 años. Considerando como punto de corte un PSA de $0.4 \mathrm{ng} / \mathrm{ml}$, un $33 \%$ de los pacientes experimentaron una recidiva clínica. $Y$ un $27 \%$ de estas recidivas ocurrieron mas allá de los 5 años de la intervención. Durante el estudio, un $29 \%$ de los pacientes con recidiva bioquímica tuvieron una recidiva clínica y el $8 \%$ fallecieron debido al tumor, con un tiempo medio actuarial entre la recidiva bioquímica y el fallecimiento de 9.8 años. El período transcurrido entre la recidiva bioquímica y la recidiva clínica no se correlacionó con el tiempo transcurrido entre la prostatectomía radical y la recidiva bioquímica. En la serie, los pacientes con un tiempo de doblaje del PSA (PSADT) inferior a 12 meses tenían un mayor riesgo de recidiva clínica.

Finalmente, en series amplias, con muchos pacientes y largo seguimiento se ha demostrado la necesidad de realizar controles más allá de los 5 años. La recidiva bioquímica puede ocurrir muchos años después de la PTR. Así, en la serie de Pound y cols. un $23 \%$ de los pacientes que tuvieron recidiva bioquímica tenían un PSA indetectable en los 5 primeros años de seguimiento y un $4 \%$ de ellos durante los 10 años de seguimiento (5).

\section{PREDICCIÓN DE LA LOCALIZACIÓN DE LA RE- CAÍDA CLÍNICA TRAS RECIDIVA BIOQUÍMICA}

Una vez detectada la recidiva bioquímica, lo más importante va a ser determinar si la recidiva clínica será local o a distancia, ya que ello determinará el tratamiento a realizar. Los principales parámetros que ayudan a diferenciar entre una y otra son los siguientes: intervalo de tiempo hasta el incremento de PSA, la velocidad del PSA, el tiempo de doblaje del PSA (PSADT), el estadío patológico y el Gleason de la pieza quirúrgica (Tabla IV) (1).

\section{Cinética del PSA}

En general, la elevación del PSA dentro de los dos primeros años de seguimiento está asociado a metástasis a distancia (11). Inicialmente Partín y cols. determinaron que la velocidad de PSA al año permitía distinguir entre recidiva local o metástasis a distancia, ya que cuando era superior a $0.75 \mathrm{ng} / \mathrm{ml} /$ año se asociaba a un mayor riesgo de enfermedad a distancia (12). Esta capacidad de diferenciación entre recidiva local y metástasis a distancia aumentaba si se combinaba con el Gleason o el estadio patológico. De acuerdo a su serie, pacientes con afectación de vesículas seminales, Gleason $\geq 8$ o afectación linfática tenían un riesgo de desarrollar enfermedad a distancia del $86 \%, 95 \%$ y $100 \%$ respectivamente. También para Lange y cols. una velocidad de PSA 
inferior a $0.75 \mathrm{ng} / \mathrm{ml} /$ año fue observada en el $94 \%$ de los pacientes con recaída local, mientras que el $56 \%$ de los pacientes con metástasis a distancia tenían un PSA superior a $0.75 \mathrm{ng} / \mathrm{ml} /$ año (13).

\section{Tiempo de doblaje del PSA}

En la actualidad se cree que la cinética del PSA durante el seguimiento es un importante factor de predicción de la recidiva clínica. Su cálculo se realiza a partir de una fórmula logarítmica que expresa el crecimiento exponencial biológico. Se calcula el PSADT a partir del logaritmo natural de 2 (0.693) dividido por la curva de la relación entre el logaritmo del PSA y el tiempo transcurrido entre las mediciones del PSA (5). Trapasso y cols. han demostrado con un PSADT de 4.3 meses esta asociado con una metástasis a distancia y una media de PSADT de $11.7 \mathrm{ng} / \mathrm{ml}$ predice un fallo local (14).
Pound y cols. revisan 1997 pacientes intervenidos de adenocarcinoma de próstata clínicamente localizado con un seguimiento medio de 5.3 años (5). Se consideró recidiva bioquímica un PSA superior a $0.2 \mathrm{ng} / \mathrm{ml}$. La supervivencia libre de metástasis de la serie fue del $82 \%$ (intervalo de confianza 95\%: 76$88 \%$ ). De los 1997 pacientes, un 15\% desarrollaron una recidiva bioquímica. De los 304 pacientes con recidiva bioquímica (que no recibieron tratamiento hormonal), un $34 \%$ desarrollaron metástasis a distancia durante el seguimiento. En este grupo, el tiempo medio actuarial hasta desarrollar una metástasis fue de 5 años (mediana 8 años) desde la elevación del PSA. En el análisis de supervivencia, el Gleason ( $\geq 8)$, el tiempo hasta la recidiva bioquímica ( $\leq 2$ años), y el PSADT (<10 meses) fueron factores predictivos para la probabilidad de desarrollar una metástasis. Pacientes con un Gleason menor a 8 tenían un $73 \%$ de probabilidad de no presentar progresión a los 5 años de

\section{TABLA IV. IMPORTANCIA CLIINICA DE LOS PARÁMETROS QUE PREDICEN LA RECIDIVA LOCAL O A DISTANCIA} TRAS LA PROSTATECTOMÍA RADICAL (1).

\begin{tabular}{|l|c|c|}
\hline $\begin{array}{l}\text { Parámetro } \\
\text { Intervalo hasta incremento PSA }\end{array}$ & Recidiva local * & Metástasis a distancia* \\
\hline$\leq 1$ año & $7 \%$ & $93 \%$ \\
\hline $1-2$ años & $10 \%$ & $90 \%$ \\
\hline $2-3$ años & $61 \%$ & $39 \%$ \\
\hline$>3$ años & $74 \%$ & $26 \%$ \\
\hline PSADT & 11.7 meses & 4.3 meses \\
\hline Gleason & & $0 \%$ \\
\hline $2-4$ & $55 \%$ & $45 \%$ \\
\hline $5-6$ & $39 \%$ & $61 \%$ \\
\hline 7 & $11 \%$ & $89 \%$ \\
\hline $8-10$ & & $84 \%$ \\
\hline Estadio patológico & $40 \%$ & $93 \%$ \\
\hline$\leq p T 2 b$ & $54 \%$ & $60 \%$ \\
\hline pT3a, RO & $48 \%$ & $52 \%$ \\
\hline pT3a, R1 & $16 \%$ & \\
\hline pT3b & $7 \%$ & \\
\hline pTxpN1 & & \\
\hline
\end{tabular}

* Intervalo de confianza 95\% 
la recidiva bioquímica, en comparación con un $40 \%$ de probabilidad en pacientes con un Gleason de 8 a 10. Pacientes con Gleason inferior a 8 y con un tiempo de recidiva del PSA superior a 2 años tenían un porcentaje del $82 \%$ de estar libre de metástasis a los 5 años, mientras que con el mismo Gleason, si el tiempo de recidiva era inferior a los dos años, la probabilidad descendía al $62 \%$. Finalmente, para pacientes con un Gleason inferior a 8 y un tiempo de recidiva menor a dos años, un PSADT superior a 10 meses suponía una probabilidad de permanecer libre de metástasis del $76 \%$ (a los 5 años), mientras que si el PSADT era inferior a 10 meses, la probabilidad descendía al 35\%.

Una vez desarrollada la metástasis, la mediana de tiempo hasta la muerte fue de 5 años. En estos casos, el tiempo entre la cirugía radical y la aparición de la metástasis fue un factor predictivo del tiempo hasta la mortalidad cáncer-específica. Los pacientes que presentaron metástasis a distancia en los 3 primeros años fallecieron antes. Los pacientes que tardaron 8 años en presentar la metástasis tuvieron 5 años más de vida.

\section{Anatomía patológica}

En estudios multivariantes sobre factores pronósticos se ha demostrado que la puntuación de Gleason es un excelente factor predictivo de la recidiva bioquímica. Así, para Chan y cols., la presencia de un Gleason de 4 o un total superior a 7 predice mal pronóstico y se considera un factor de alto riesgo para una BCR (15).

De acuerdo a las "Guías de la Asociación Europea de Urología", la recidiva local se predice con un $80 \%$ de probabilidad por una elevación del PSA tras de 3 años de la PTR, un PSADT superior a 11 meses, un Gleason $\leq 6$ y un estadio $\leq \mathrm{pT} 3 a$ pNO - $\mathrm{pTXR} 1$. Por otra parte, la metástasis a distancia se predicen con un $80 \%$ de probabilidad con una elevación del PSA a menos de 1 año tras la PTR, con un PSADT de 4-6 meses, un Gleason 8-10 o un estadio patológico $\mathrm{pT} 3 \mathrm{~b}$ o $\mathrm{pTx} \mathrm{pN} 1$ (1).

\section{SIGNIFICADO PRONÓSTICO DE LA RECIDIVA BIOQUÍMICA}

\section{Recidiva bioquímica}

Para algunos autores, no existen diferencias de supervivencia global en función de la recidiva bioquímica. Así, Jhaveri y cols. estudian 1132 pacientes tras PTR (16). Con un seguimiento medio de 56 meses, el 19\% de los pacientes presentaron una recidiva bioquímica (PSA superior a $0.2 \mathrm{ng} / \mathrm{ml}$ ). La supervivencia global a los 10 años fue similar en los pacientes con recidiva bioquímica (88\%) que en aquellos que no la presentaron (93\%).

Dado que la recidiva bioquímica per se puede no ser suficiente como factor pronóstico, se ha propuesto implicar otros factores como el PSADT y las características anatomopatológicas de la pieza quirúrgica para obtener mayor información pronóstica.

\section{Tiempo de doblaje del PSA (PSADT)}

Muchos autores consideran que el tiempo de doblaje del PSA (PSADT) está muy relacionado con la supervivencia cáncer específica tras PTR $(17,18)$.

Albertsen y cols. consideran que un PSADT inferior al año incrementa el riesgo de mortalidad cáncer especifica en los siguientes diez años en pacientes sometidos a una PTR o a radioterapia radical (19). Tras analizar 1136 pacientes, el PSADT medio de aquellos que fallecieron por cáncer de próstata fue de 0.8 años, mientras que aquellos que no fallecieron durante el seguimiento de 10 años no habían tenido recidiva bioquímica o su PSADT fue superior a 1 año.

En un estudio muy extenso de 8669 pacientes tratados con prostatectomía radical o radioterapia radical, D'Amico demuestra que el PSADT está relacionado con la mortalidad cáncer específica y la mortalidad global (20). Para D'Amico, el PSADT inferior a 3 meses es un factor de mal pronóstico.

\section{Anatomía patológica y PSADT}

Freedland y cols. evalúan 379 pacientes sometidos a PTR con BCR (mediana de seguimiento de 10) (21). La recidiva bioquímica fue considerada con un PSA postoperatorio superior a $0.2 \mathrm{ng} / \mathrm{ml}$. En el análisis multivariante, el Gleason ( $\leq 7$ vs. 8-19), el tiempo hasta la recidiva bioquímica ( $\leq 3$ vs. $>3$ años) y el PSADT fueron los factores de riesgo para la mortalidad cáncer-específica después de BCR. Usando estos 3 parámetros, estos autores estratifican los pacientes en grupos de alto y bajo riesgo de mortalidad. Pacientes con un PSADT inferior a 3 meses, un tiempo de BCR tras la PTR inferior a 3 años y un Gleason 810 tienen una mediana de supervivencia de 3 años. Por el contrario, pacientes con un PSADT superior a 15 meses y un tiempo hasta la BCR superior a 3 años tienen una supervivencia del $100 \%$.

La probabilidad de supervivencia libre de recidiva bioquímica se relaciona de forma inversa con el estadio patológico de la enfermedad, siendo los 
TABLA V. PROBABILIDAD DE RECIDIVA BIOQUIIMICA A LOS 5 Y 10 AÑOS DE SEGUIMIENTO EN FUNCIÓN DEL ESTADIO PATOLÓGICO, GLEASON Y MÁRGENES QUIRÚRGICOS (42).

\begin{tabular}{|c|c|c|c|c|c|}
\hline Estadio patológico & Gleason & Márgen quirúrgico & $\begin{array}{l}\text { Supervivencia } \\
\text { libre BCR } \\
5 \text { años* }\end{array}$ & $\begin{array}{l}\text { Supervivencia } \\
\text { libre BCR } \\
10 \text { años* }\end{array}$ & Grupo riesgo \\
\hline OC ○ EEP & $2-6$ & Negativo & $97(95-98)$ & $95(92-96)$ & Excelente \\
\hline OC ○ EEP & 7 & Negativo & $86(82-90)$ & $72(62-80)$ & \multirow[t]{2}{*}{ Bueno } \\
\hline OC ○ EEP & $2-6$ & Positivo & & & \\
\hline OC & $8-10$ & Positivo/negat. & $62(51-70)$ & $41(29-55)$ & \multirow[t]{4}{*}{ Moderado } \\
\hline EEP & $8-10$ & Negativo & & & \\
\hline EEP & $7-10$ & Positivo & & & \\
\hline VVSS + y N- & $2-10$ & Positivo/negat. & & & \\
\hline $\mathrm{N}+$ & $2-10$ & Positivo/negat. & $37(26-48)$ & $13(4-26)$ & Bajo \\
\hline
\end{tabular}

OC= Órgano-Confinado EEP $=$ Extensión Extraprostática WSS = Vesículas Seminales
$N=$ Ganglios Linfáticos

$B C R=$ Fracaso o Recidiva Bioquímica

* Probabilidad e intervalo de confianza 95\%

\section{TABLA VI. REGRESIÓN DE COX DE LOS FACTORES QUE PREDICEN LA PROGRESIÓN DEL PSA TRAS RADIOTERAPIA POST-PROSTATECTOMÍA RADICAL (22).}

\begin{tabular}{|l|c|c|}
\hline Factor pronóstico & Recidiva local ${ }^{*}$ & $P$ \\
\hline PSA preprostatectomía $>10 \mathrm{ng} / \mathrm{ml}$ & $1.1(0.8-1.4)$ & 0.73 \\
\hline Gleason 4-6 & Referencia & 0.06 \\
\hline Gleason 7 & $1.5(0.98-2.2)$ & $<0.001$ \\
\hline Gleason 8-10 & $2.6(1.7-4.1)$ & \\
\hline PSA prerradioterapia $\leq 1.0 \mathrm{ng} / \mathrm{ml}$ & Referencia & 0.31 \\
\hline PSA prerradioterapia $1.0-2.0 \mathrm{ng} / \mathrm{ml}$ & $1.2(0.8-1.7)$ & $<0.001$ \\
\hline PSA prerradioterapia $>2 \mathrm{ng} / \mathrm{ml}$ & $2.3(1.7-3.2)$ & $<0.001$ \\
\hline Márgenes quirúrgicos negativos & $1.9(1.4-2.5)$ & 0.001 \\
\hline PSADT $\leq 10$ meses & $1.7(1.2-2.2)$ & 0.02 \\
\hline Afectación vesículas seminales & $1.4(1.1-1.9)$ & 0.21 \\
\hline Extensión extracapsular & $1.2(0.9-1.6)$ & 0.32 \\
\hline Ganglios positivos & $1.5(0.7-3.0)$ & 0.71 \\
\hline Intervalo libre de enfermedad <12 meses & $1.0(0.7-1.3)$ & 0.16 \\
\hline Hormonoterapia previa a radioterapia & $0.8(0.5-1.1)$ & 0.96 \\
\hline Dosis radioterapia <64.8Gy & $1.0(0.7-1.3)$ & \\
\hline
\end{tabular}


principales criterios patológicos para definir el pronóstico el grado tumoral, la infiltración de vesículas seminales y la afectación de los ganglios linfáticos (4). Con el objeto de estimar el riesgo de recidiva bioquímica tras PTR en función de los datos patológicos, Khan y cols. analizan 1955 pacientes. A partir del Gleason, estadio patológico y márgenes quirúrgicos confeccionan cuatro grupos de riesgo en función de la probabilidad de recidiva bioquímica a los 5 y 10 años de seguimiento (Tabla V).

Estos parámetros también han demostrado su validez pronóstica para predecir la recidiva bioquímica en pacientes que han recibido radioterapia de rescate tras PTR. Stephenson y cols. evaluan 501 pacientes intervenidos de prostatectomía radical que han recibido radioterapia de rescate (22). El 96\% de los pacientes tenían un PSA pre-radioterapia superior a $0.2 \mathrm{ng} / \mathrm{ml}$, con una media de $0.72 \mathrm{ng} / \mathrm{ml}(0.1-26)$. Con una media de seguimiento de 54 meses, el 50\% de los pacientes presentaron progresión de la enfermedad tras la radioterapia. La supervivencia libre de enfermedad y la supervivencia global a los siete años fueron del $90 \%$ y $82 \%$, respectivamente. En el análisis multivariante, la progresión de PSA se asoció con un Gleason $\geq 8$, un PSA pre-radioterapia inferior a $2 \mathrm{ng} / \mathrm{ml}$, márgenes quirúrgicos negativos, un PSADT inferior a 10 meses y afectación de las vesículas seminales (Tabla VI).

\section{VALORACIÓN DEL PACIENTE CON RECIDIVA BIOQUÍMICA}

\section{Biopsia y tacto}

La utilidad del tacto rectal y la biopsia del lecho quirúrgico en estos pacientes son dudosas. En relación al tacto, Obeck y cols., estudian una serie de 501 pacientes tras PTR (23). Ellos utilizan como definición de BCR la presencia de PSA superior a 0.2 $\mathrm{ng} / \mathrm{ml}$ y dos elevaciones consecutivas posteriores. Tras un seguimiento medio de 25 meses, un $14.4 \%$ de los pacientes (72 pacientes) tuvieron una BCR. Únicamente en 4 de estos 72 pacientes el tacto rectal fue patológico y en ninguno de ellos en el momento del tacto, el PSA fue indetectable.

La especificidad de la biopsia ecodirigida para la detección de la recidiva local es baja. Así, Connolly y cols. (24) evalúan 156 biopsias transrectales practicadas en 114 pacientes con valores de PSA persistentemente elevados tras una PTR. De ellos, sólo en el $54 \%$ de los casos se diagnosticó una recidiva, con una media de PSA de $5.7 \mathrm{ng} / \mathrm{ml}$. La sensibilidad diagnóstica fue el $91.9 \%$, la especificidad del $29.1 \%$, y el valor predictivo positivo y negativo tan sólo del $66.9 \%$ y $69.6 \%$, respectivamente. Por lo tanto, únicamente en los casos en los que se aprecia una lesión palpable o un nódulo hipoecoico en la ecografía transrectal estaría justificado realizar una biopsia ecodirigida.

\section{Exploraciones complementarias}

El objetivo de las exploraciones complementarias ante una recidiva bioquímica es intentar determinar la presencia de recidiva clínica local o a distancia para indicar el tratamiento más adecuado. Sin embargo, el rendimiento de las exploraciones complementarias en pacientes con recidiva bioquímica es muy bajo (2). Las dos pruebas por excelencia en estos casos son la gammagrafía ósea y el TAC abdominopélvico. No obstante, también se recomienda realizar una radiografía de tórax para descartar las metástasis pulmonares. En caso de dudas en la gammagrafia ósea se debe realizar una radiografía simple de la zona problema (4).

\section{TAC abdominopélvico y gammagrafía ósea}

Kane y cols. valoran 131 pacientes con recidiva bioquímica a los que se les practica TAC abdominopélvico y gammagrafía ósea durante un seguimiento medio de 3 años (25). De ellos, sólo en el $9.4 \%$ la gammagrafía fue positiva. La media del PSA en el momento de la gammagrafía positiva fue de $61.3 \mathrm{ng} / \mathrm{ml}$. Únicamente dos pacientes con la gammagrafía ósea positiva tuvieron una velocidad de PSA inferior a 0.5 $\mathrm{ng} / \mathrm{ml} / \mathrm{mes}$. Por otra parte, sólo un $14 \%$ tuvieron un TAC abdominal positivo. En este estudio, tanto en el TAC como en la gammagrafía el factor predictivo más importante fue la velocidad del PSA.

Cher y cols. realizan el seguimiento de 122 pacientes tratados con PTR y con recidiva bioquímica (26). Durante el seguimiento, únicamente el $4.1 \%$ de los mismos tuvo una gammagrafía positiva. En su caso, el valor de PSA más bajo asociado a una gammagrafia ósea positiva fue de $46 \mathrm{ng} / \mathrm{ml}$. Los únicos factores predictivos en el análisis multivariante fueron el PSA total y la velocidad de PSA. En esta serie, valores de PSA inferiores a $40 \mathrm{ng} / \mathrm{ml}$ y velocidades de PSA inferiores a $5 \mathrm{ng} / \mathrm{ml} / \mathrm{mes}$ se asociaban una probabilidad menor del $5 \%$ para una gammagrafía positiva.

Para Okotie y cols., los pacientes con un PSADT inferior a 6 meses tenían un riesgo mayor de tener una gammagrafía ( $26 \%$ frente a $3 \%$ ) o TAC positivo $(24 \%$ frente a $0 \%)$ frente a aquellos con un PSADT superior (27). En los pacientes con PSADT inferior a 6 meses, el riesgo de tener una exploración positiva dependía principalmente del nivel de PSA en el momento de realizar la exploración. Para pa- 
cientes con PSA inferior a $10 \mathrm{ng} / \mathrm{ml}$, la posibilidad de tener un TAC o una gammagrafía positiva era del $0 \%$ y del $11 \%$, respectivamente. Sin embargo, para PSA mayores a $10 \mathrm{ng} / \mathrm{ml}$ los riesgos para un TAC o una gammagrafía positiva eran del $57 \%$ y $46 \%$, respectivamente.

Actualmente, las "Guías Clínicas de la Asociación Europea de Urología" recomiendan no realizar el TAC y la gammagrafía ósea en pacientes con PSA inferior a $20 \mathrm{ng} / \mathrm{ml}$ o una velocidad de PSA inferior a $20 \mathrm{ng} / \mathrm{ml} /$ año (1).

\section{ProstaScint Scan $®$}

La inmunogammagrafía es una técnica en la que se emplea un anticuerpo monoclonal marcado frente al antígeno prostático de membrana (PMSA). Este antígeno es una proteína de membrana que se expresa en todas las células del cáncer de próstata y en algunos casos, en el epitelio de la hiperplasia benigna. Prostascint $®$ (Prostascint, Cytogen Corporation, Princenton, NJ) emplea un anticuerpo monoclonal marcado ( ${ }^{111}$ l-7E 1 1) frente al antígeno prostático de membrana. El problema es que este anticuerpo marcado se liga a un epítopo intracelular de la molécula de PMSA, por lo que sólo las células muertas o en vías de morir expresan PMSA. No obstante, y a pesar de estas dificultades, se ha publicado hasta un $81 \%$ de rendimiento diagnóstico en la localización de la recidiva en pacientes con BCR (1).

Raj y cols. han demostrado la utilidad de la inmunogammagrafia con ${ }^{111} / n$-capromab pendetide en pacientes con recidiva bioquímica y PSA $\leq 4 \mathrm{ng} /$ $\mathrm{ml}(28)$. Estos autores evidenciaron captación positiva del isótopo en un $72 \%$ de los 255 pacientes con BCR. De ellos, en un $31 \%$ de los casos se trataba de una recidiva local, en un $42 \%$ de recidiva locorregional y en un $25 \%$ de metástasis a distancia. La inmunogammagrafia positiva no se correlacionó con el Gleason, estadio patológico, invasión perineural o márgenes positivos. Por el contrario, únicamente el $12 \%$ y el $16 \%$ de los pacientes presentaron un gammagrafía ósea o un TAC positivo, respectivamente.

\section{Tomografía de emisión de positrones}

Actualmente, el estudio con tomografia de emisión de positrones (PET) no ofrece el rendimiento necesario para el estudio de pacientes con recidiva bioquímica. Sin embargo, algunos estudios son esperanzadores. Kotzerke y cols. han demostrado que la realización de un PET con "-Carbono acetato fue positivo en 15 de 18 pacientes con biopsia positiva de recidiva local y no hubo ningún falso positivo en pacientes con biopsia negativa (29).

\section{Resonancia nuclear magnética (RMN)}

La resonancia magnética se considera comparable al TAC a la hora de detectar metástasis ganglionares (4). Los mejores resultados con la resonancia se consiguen utilizando un coil endorrectal. Sella y cols. valoraron retrospectivamente 48 pacientes con recidiva local clínicamente demostrada, en los cuales la RMN demostró recidiva local en 39 de ellos (81\%). La media del PSA en el momento del diagnóstico fue de $2 \mathrm{ng} / \mathrm{ml}$. Las recidivas se localizaron principalmente a nivel retrovesical $(40 \%)$ y perianastomótico (29\%) (30). Por otra parte y como apuesta de futuro, Harisinghani y cols. han demostrado que la RMN de alta resolución con nanoparticulas magnéticas linfotrópicas mejoran la sensibilidad en la detección de metástasis ganglionares (31).

\section{TRATAMIENTO ANTE UNA RECIDIVA BIOQUI- MICA TRAS PROSTATECTOMÍA RADICAL}

Las posibilidades de tratamiento ante el fracaso bioquímico de una PTR están sometidas todavía a debate. Las posibilidades terapéuticas incluyen la radioterapia del lecho quirúrgico, el bloqueo hormonal, la combinación de antiandrógenos con inhibidores de la $5 \alpha$-reductasa o la quimioterapia. (1).

\section{Radioterapia de rescate}

Actualmente se considera que los pacientes con una recidiva bioquímica sin evidencia radiológica de metástasis a distancia son candidatos ideales para el tratamiento local con radioterapia. Recientemente, Macdonald y cols. han valorado la supervivencia libre de de recaída bioquímica y supervivencia global en pacientes que fueron tratados por recidiva bioquímica o por recidiva clínica (tacto rectal) (32). En el primer caso la supervivencia libre de recidiva bioquímica y supervivencia global del primer grupo fue de $69 \%$ y $96 \%$, frente al $45 \%$ y $78 \%$ del segundo grupo

EI PSA pretratamiento es el principal factor relacionado con el éxito del tratamiento. Wu y cols. y Schild y cols. indican un punto de corte de $2.5 \mathrm{ng} / \mathrm{ml}$. Así, estos autores refieren una tasa de supervivencia libre de enfermedad del $53 \%$ y $76 \%$ para pacientes con PSA inferior a este punto, frente a tasas muy bajas si se retarda el inicio del tratamiento por encima de ese nivel ( $8 \%$ y $26 \%$, respectivamente) $(33,34)$. Con niveles de PSA de $2.0 \mathrm{ng} / \mathrm{ml}$, Forman y cols. refieren una tasa de supervivencia libre de enfermedad del $83 \%$ para valores inferiores y del $33 \%$ para valores superiores (35). También para Stephenson y cols. el PSA preoperatorio superior a $2.0 \mathrm{ng} / \mathrm{ml}$ está 
asociado a un mayor riesgo de progresión en el análisis multivariante (22). Actualmente, el documento de consenso de la ASTRO recomienda tratar a pacientes con un PSA post-prostatectomía radical inferior a 1.5 $\mathrm{ng} / \mathrm{ml}$ aplicando una dosis de $64 \mathrm{~Gy}$ (36).

Para Stephenson y cols., la presencia de márgenes quirúrgicos positivos es un factor pronóstico de la respuesta a la radioterapia post-PTR (22). Para este autor, la recidiva de la enfermedad en presencia de márgenes positivos suele corresponder a una recidiva local, aun en presencia de factores que sugieren la recidiva sistémica como un Gleason elevado o un PSADT bajo. Para este autor, estos dos factores pronósticos asociados habitualmente a recidiva a distancia no pueden contraindicar la realización de la radioterapia de rescate. De acuerdo a sus resultados, el $51 \%$ de los pacientes con un Gleason 8-10 y márgenes positivos conseguían una respuesta prolongada a la radioterapia de rescate (4 años libres de recidiva bioquímica con PSA inferior a $0.1 \mathrm{ng} / \mathrm{ml}$ ). Es mas, un $37 \%$ de los pacientes de este grupo (Gleason 8-10 y márgenes positivos) con un PSADT inferior a 10 meses estaban libres de recidiva bioquímica a los 4 años.

\section{Tratamiento hormonal}

La utilidad del tratamiento hormonal ante la recidiva bioquímica es un tema sometido a debate. Para los pacientes sin metástasis a distancia y ganglios positivos, parece ser que la hormonoterapia precoz puede tener un efecto beneficioso sobre las metástasis ocultas a distancia (1). Si hay evidencias de que el bloqueo hormonal puede tener efectos sobre la enfermedad metastásica mínima $(37,38)$, esta debería tener el mismo efecto sobre la recidiva bioquímica. No obstante, habría que valorar también los posibles efectos secundarios, como la osteoporosis, la pérdida de masa muscular, los sofocos o la disminución de la líbido.

Moul y cols. estudian retrospectivamente 1352 pacientes con recidiva bioquímica tras PTR, observando un retraso en el tiempo de aparición de metástasis en el grupo de pacientes de alto riesgo (Gleason mayor de 7 o PSADT inferior a 12 meses) con hormonoterapia adyuvante (hormonoterapia precoz). No obstante, este beneficio no se traslada posteriormente a la supervivencia global de la serie (39).

Con el objeto de reducir los efectos secundarios de la hormonoterapia se ha propuesto la monoterapia con antiandrógenos. De acuerdo con Wirth y cols., el tratamiento con bicalutamida a dosis diaria de $150 \mathrm{mg}$ reduce el riesgo de progresión tumoral en pacientes sometidos a radioterapia o prostatectomía radical (40). El estudio randomizado inicial incluía 3603 pacientes con una media de seguimiento de 2.6 años, en los que se demostraba una reducción del riesgo de progresión del PSA, así como también una disminución del riesgo de progresión clínica. Recientemente, Mcleod y cols. han publicado datos con mayor número de pacientes y seguimiento (41). Se trata de 8113 pacientes con cáncer de próstata localizado (T1-2, NO/NX) o localmente avanzado (T3-4, cualquier $\mathrm{N}$; o cualquier $\mathrm{T}$ con ganglios positivos) sin metástasis a distancia. Estos pacientes fueron tratados mediante prostatectomía radical, radioterapia radical o conducta expectante. Con una media de seguimiento de 7.4 años, no se ha comprobado que la hormonoterapia adyuvante con bicalutamida prolongue la supervivencia en el subgrupo de pacientes tratados inicialmente con PTR.

Actualmente y de acuerdo a las "Guías Clínicas de la Asociación Europea de Urología", en pacientes con un elevado PSA preoperatorio ( $>20$ $\mathrm{ng} / \mathrm{ml}$ ), Gleason $\geq 7$, extensos márgenes quirúrgicos positivos y crecimiento extraprostático (pT3b, $\mathrm{pTxpN} 1$ ) podría estar indicado, a pesar de que no se ha ratificado su utilidad, el tratamiento hormonal adyuvante (1).

\section{CONCLUSIONES}

Dada la facilidad de la monitorización del PSA y la poca rentabilidad diagnóstica del tacto rectal y la biopsia del lecho prostático, este valor se ha convertido en la herramienta fundamental en el seguimiento de la prostatectomía radical. Así, el concepto de recidiva bioquímica está desplazando al de recidiva clínica a la hora de decidir una segunda línea de tratamiento.

No obstante, uno de los problemas de la recidiva bioquímica es la determinación del nivel de corte para su aplicación. Se trata de encontrar el equilibrio entre el punto más bajo (para garantizar el diagnóstico precoz), y aquel que permita descartar los pacientes que no van a desarrollar nunca una recidiva clínica (para evitar el sobre-tratamiento). En la actualidad, el punto de corte con mayor consenso para el diagnóstico de una recidiva bioquímica es de $0.4 \mathrm{ng} / \mathrm{ml}$.

Sin embargo, en los últimos años, al concepto puramente "estático" del nivel de PSA, ha sido sobrepasado por una visión más "longitudinal", con la incorporación del tiempo de doblaje del PSA. En general, un paciente con una elevación progresiva del PSA y un PSADT inferior a 10 meses tiene una 
alta probabilidad de desarrollar una metástasis a distancia durante el seguimiento, mientras que si este valor es superior a 10 meses, habría que pensar en una probable recidiva local.

Además de herramienta para la ubicación de una futura recidiva clínica, el PSADT tiene implicaciones pronósticas per se, ya que se ha demostrado que un valor inferior a 3 meses implica un aumento de la mortalidad cáncer-específica. No obstante, en este momento es mejor combinar este dato con otros indicadores, como es el lapso de tiempo entre la prostatectomía radical y la recidiva bioquímica (peor pronóstico cuando es inferior a 3 años) y la puntuación Gleason de la pieza quirúrgica (peor pronóstico para valores iguales o superiores a 8 ).

Dada la importancia clínica que ha adquirido el valor del PSA en el seguimiento, en la actualidad está indicado realizar radioterapia de rescate ante una recidiva bioquímica, sin la necesidad de confirmación histológica. Así, se debe indicar radioterapia del lecho quirúrgico (64Gy) en aquellos pacientes con un PSA postoperatorio no superior a $1.5 \mathrm{ng} / \mathrm{ml}$. En estos casos, la realización de un TAC abdomino-pélvico o una gammagrafía ósea no está indicado, ya que estas pruebas no han demostrado una buena rentabilidad diagnóstica con valores de PSA inferiores a $20 \mathrm{ng} / \mathrm{ml}$.

\section{BIBLIOGRAFÍA Y LECTURAS RECOMENDADAS ( ${ }^{*}$ lectura de interés $y^{* *}$ lectura fundamental)}

1. 1. AUS, G.; ABBOU, C.C.; BOLLA, M. y cols.: "Guidelines on Prostate Cancer". Guidelines, ed. European Association of Urology. Arnhem: EAU Guidelines Office, chapt 1-106, 2006.

*2. WARD, J.F.; MOUL, J.W.: "Biochemical recurrence after definitive prostate cancer therapy. Part I: defining and localizing biochemical recurrence of prostate cancer". Curr. Opin. Urol., 15: $181,2005$.

3. PARTIN, A.W.; CARTER, H.B.; CHAN, D.W. y cols.: "Prostate specific antigen in the staging of localized prostate cancer: influence of tumor differentiation, tumor volume and benign hyperplasia”. J. Urol., 143: 747, 1990.

4. KHAN, M.A.; PARTIN, A.W.: "Management of patients with an increasing prostate-specific antigen after radical prostatectomy". Curr. Urol. Rep., 5: 179, 2004.

**5. POUND, C.R.; PARTIN, A.W.; EISENBERGER, M.A. y cols.: "Natural history of progression after PSA elevation following radical prostatectomy". JAMA, 281: 1591, 1999.
**6. AMLING, C.L.; BERGSTRALH, E.J.; BLUTE, M.L. y cols.: "Defining prostate specific antigen progression after radical prostatectomy: what is the most appropriate cut point?". J. Urol., 165: $1146,2001$.

**7. FREEDLAND, S.J.; SUTTER, M.E.; DOREY, F. y cols.: "Defining the ideal cut point for determining PSA recurrence after radical prostatectomy. Prostate-specific antigen". Urology, 61: 365, 2003.

8. STEPHENSON, A.J.; SCARDINO, P.T.; EASTHAM, J.A. y cols.: "Defining biochemical recurrence of prostate cancer after radical prostatectomy: a proposal for a standardized definition". J. Urol., 173, 2005.

9. AMERICAN SOCIETY FOR THERAPEUTIC RADIOLOGY AND ONCOLOGY CONSENSUS PANEL.: "Consensus statement: guidelines for PSA following radiation therapy. American Society for Therapeutic Radiology and Oncology Consensus Panel”. Int. J. Radiat. Oncol. Biol. Phys., 37: 1035, 1997.

10. WARD, J.F.; BLUTE, M.L.; SLEZAK, J. y cols.: "The long-term clinical impact of biochemical recurrence of prostate cancer 5 or more years after radical prostatectomy". J. Urol., 170: 1872, 2003.

11. PARTIN, A.W.; OESTERLING, J.E.: "The clinical usefulness of prostate specific antigen: update 1994”. J. Urol., 152: 1358, 1994.

12. PARTIN, A.W.; PEARSON, J.D.; LANDIS, P.K. y cols.: "Evaluation of serum prostate-specific antigen velocity after radical prostatectomy to distinguish local recurrence from distant metastases". Urology, 43: 649, 1994.

13. LANGE, P.H.; ERCOLE, C.J.; LIGHTNER, D.J. y cols.: "The value of serum prostate specific antigen determinations before and after radical prostatectomy". J. Urol., 141: 873, 1989.

14. TRAPASSO, J.G.; DEKERNION, J.B.; SMITH, R.B. y cols.: "The incidence and significance of detectable levels of serum prostate specific antigen after radical prostatectomy". J. Urol., 152: $1821,1994$.

15. CHAN, T.Y.; PARTIN, A.W.; WALSH, P.C. y cols.: "Prognostic significance of Gleason score $3+4$ versus Gleason score $4+3$ tumor at radical prostatectomy". Urology, 56: 823, 2000.

16. JHAVERI, F.M.; ZIPPE, C.D.; KLEIN, E.A. y cols.: "Biochemical failure does not predict overall survival after radical prostatectomy for localized prostate cancer: 10-year results". Urology, 54: 884, 1999.

17. ROBERTS, S.G.;BLUTE, M.L.;BERGSTRALH, E.J.: "PSA doubling time as a predictor of clinical progression after biochemical failure following radical prostatectomy for prostate cancer". Mayo Clin. Proc., 76: 576, 2001.

18. PRUTHI, R.S.; JOHNSTONE, I.; TU, I.P. y cols.: "Prostate-specific antigen doubling times in pa- 
tients who have failed radical prostatectomy: correlation with histologic characteristics of the primary cancer". Urology, 49: 737, 1997.

19. ALBERTSEN, P.C.; HANLEY, J.A.; PENSON, D.F. y cols.: "Validation of increasing prostate specific antigen as a predictor of prostate cancer death after treatment of localized prostate cancer with surgery or radiation". J. Urol., 171: 2221, 2004.

20. D'AMICO, A.V.; MOUL, J.W.; CARROLL, P.R. y cols.: "Surrogate end point for prostate cancerspecific mortality after radical prostatectomy or radiation therapy". J. Natl. Cancer Inst., 95: 1376, 2003.

*21. FREEDLAND, S.J.; HUMPHREYS, E.B.; MANGOLD, L.A. y cols.: "Risk of prostate cancer-specific mortality following biochemical recurrence after radical prostatectomy". JAMA, 294: 433, 2005.

**22. STEPHENSON, A.J.; SHARIAT, S.F.; ZELEFSKY, M.J. y cols.: "Salvage radiotherapy for recurrent prostate cancer after radical prostatectomy". JAMA, 291: 1325, 2004.

23. OBEK, C.; NEULANDER, E.; SADEK, S. y cols.: "Is there a role for digital rectal examination in the follow up of patients after radical prostatectomy?". J. Urol., 162: 762, 1999.

24. CONNOLLY, J.A.; SHINOHARA, K.; PRESTI, J.C. y cols.: "Local recurrence after radical prostatectomy: characteristics in size, location, and relationship to prostate-specific antigen and surgical margins". Urology, 47: 225, 1996.

25. KANE, C.J.; AMLING, C.L.; JOHNSTONE, P.A. y cols.: "Limited value of bone scintigraphy and computed tomography in assessing biochemical failure after radical prostatectomy". Urology, 61: 607, 2003.

26. CHER, M.L.; BIANCO, F.J. Jr.; LAM, J.S. y cols.: "Limited role of radionuclide bone scintigraphy in patients with prostate specific antigen elevations after radical prostatectomy". J. Urol., 160: 1387, 1998.

27. OKOTIE, O.T.; ARONSON, W.J.; WIEDER, J.A. y cols.: "Predictors of metastatic disease in men with biochemical failure following radical prostatectomy". J. Urol., 171: 2260, 2004.

28. RAJ, G.V.; PARTIN, A.W.; POLASCIK, T.J.: "Clinical utility of indium 111-capromab pendetide immunoscintigraphy in the detection of early, recurrent prostate carcinoma after radical prostatectomy". Cancer, 94: 987, 2002.

29. KOTZERKE, J.; VOLKMER, B.G.; NEUMAIER, B. y cols.: "Carbon-11 acetate positron emission tomography can detect local recurrence of prostate cancer". Eur. J. Nucl. Med. Mol. Imaging., 29: 1380, 2002.

30. SELLA, T.; SCHWARTZ, L.H.; SWINDLE, P.W. y cols.: "Suspected local recurrence after radical prostatectomy: endorectal coil MR imaging”. Radiology, 231: 379, 2004.
31. HARISINGHANI, M.G.; BARENTSZ, J.; HAHN, P.F. y cols.: "Noninvasive detection of clinically occult lymph-node metastases in prostate cancer”. N. Engl. J. Med., 348: 2491, 2003.

32. McDONALD, O.K.; SCHILD, S.E.; VORA, S. y cols.: "Salvage radiotherapy for men with isolated rising PSA or locally palpable recurrence after radical prostatectomy: do outcomes differ?". Urology, 64: 760, 2004.

33. WU, J.J.; KING, S.C.; MONTANA, G.S. y cols.: "The efficacy of postprostatectomy radiotherapy in patients with an isolated elevation of serum prostate-specific antigen". Int. J. Radiat. Oncol. Biol. Phys., 32: 317, 1995.

34. SCHILD, S.E.; BUSKIRK, S.J.; WONG, W.W. y cols.: "The use of radiotherapy for patients with isolated elevation of serum prostate specific antigen following radical prostatectomy". J. Urol., 156: $1725,1996$.

35. FORMAN, J.D.; MEETZE, K.; PONTES, E. y cols.: "Therapeutic irradiation for patients with an elevated post-prostatectomy prostate specific antigen level". J. Urol., 158: 1436, 1997.

36. COX, J.D.; GALLAGHER, M.J.; HAMMOND, E.H. y cols.: "Consensus statements on radiation therapy of prostate cancer: guidelines for prostate re-biopsy after radiation and for radiation therapy with rising prostate-specific antigen levels after radical prostatectomy. American Society for Therapeutic Radiology and Oncology Consensus Panel". J. Clin. Oncol., 17: 1155, 1999.

37. CRAWFORD, E.D.; EISENBERGER, M.A.; McLEOD, D.G. y cols.: "A controlled trial of leuprolide with and without flutamide in prostatic carcinoma”. N. Engl. J. Med., 321: 419, 1989.

38. DENIS, L.J.; KEUPPENS, F.; SMITH, P.H. y cols.: "Maximal androgen blockade: final analysis of EORTC phase III trial 30853. EORTC Genito-Urinary Tract Cancer Cooperative Group and the EORTC Data Center". Eur. Urol., 33: 144, 1998.

39. MOUL, J.W.; WU, H.; SUN, L. y cols.: "Early versus delayed hormonal therapy for prostate specific antigen only recurrence of prostate cancer after radical prostatectomy". J. Urol., 171: 1141, 2004.

40. WIRTH, M.: "Delaying/reducing the risk of clinical tumour progression after primary curative procedures". Eur. Urol., 2: 17, 2001.

41. McLEOD, D.G.; IVERSEN, P.; SEE, W.A. y cols.: "Bicalutamide $150 \mathrm{mg}$ plus standard care vs standard care alone for early prostate cancer". BJU Int., 97: 247, 2006.

*42. KHAN, M.A.; PARTIN, A.W.; MANGOLD, L.A. y cols.: "Probability of biochemical recurrence by analysis of pathologic stage, Gleason score, and margin status for localized prostate cancer". Urology, 62: 866, 2003. 